\title{
Breast Tumor Computer-aided Diagnosis using Self-Validating Cerebellar Model Neural Networks
}

\author{
Jian-sheng Guan ${ }^{1,4}$, Lo-Yi Lin ${ }^{2}$, Guo-li Ji ${ }^{1}$, Chih-Min Lin ${ }^{3,4,5, *}$, \\ Tien-Loc Le ${ }^{5}$, Imre J. Rudas ${ }^{6}$ \\ ${ }^{1}$ Department of Automation, Xiamen University, Xiamen 361000, China \\ jsguan@xmut.edu.cn, glji@xmu.edu.cn \\ ${ }^{2}$ School of Medicine, Taipei Medical University, Taipei 100, Taiwan \\ b101098025@tmu.edu.tw
}
${ }^{3}$ School of Information Science and Engineering, Xiamen University, Xiamen 361000, China,cml@saturn.yzu.edu.tw
${ }^{4}$ College of Electrical Engineering and Automation, Xiamen University of Technology, Xiamen 361024, China, jsguan@xmut.edu.cn
5* Corresponding author, Department of Electrical Engineering and Innovation Center for Big Data and Digital Convergence, Yuan Ze University, Taoyuan 320, Taiwan,cml@saturn.yzu.edu.tw, s1038505@mail.yzu.edu.tw
${ }^{6}$ Óbuda University, H-1034 Budapest, Hungary, e-mail: rudas@uni-obuda.hu

\begin{abstract}
Breast cancer is becoming a leading cause of death among women in the world. However, it is confirmed that early detection and accurate diagnosis of this disease can ensure a long survival of the patients. This study proposes a self-validation cerebellar model articulation controller (SVCMAC) neural network which can yield high accuracy of predication and low false-negative rate for breast cancer diagnosis. With its self-validation unit, the SVCMAC neural network has higher classification accuracy than the conventional CMAC neural network. The parameters of the receptive-field basis function and the weights are all updated first by training data, and the most suitable parameters are then chosen through the self-validation algorithm to retrain the neural network for better performance. Experimental results provide evidence that the SVCMAC neural network has a higher classification accuracy when compared with the BP neural network, LVQ neural network and CMAC neural network.
\end{abstract}


Keywords: cerebellar model articulation controller; breast cancer diagnosis; selfvalidation

\section{Introduction}

Breast cancer is the leading type of cancer in women, accounting for $25 \%$ of all cases worldwide. In 2012, it resulted in 1.68 million cases and 522,000 deaths [1]. It is more prevalent in developed countries [2] and is about 100 times more common among women than men [3]. Belgium has the highest rate of breast cancer, followed by Denmark and France [4]. Therefore, prompt detection, early diagnosis and active prevention can minimize the risk of unneeded suffering from this disease.

A palpable breast lump, whether benign or malignant, is a cause of anxiety to the patient. Therefore, accurate pathological diagnosis is crucial for further treatment and estimation of an outcome [5]. The key issue in the diagnosis of breast cancer is to determine whether the lump is benign or malignant, especially for patients who are not suitable for surgery. Fine needle aspiration cytology (FNAC) has become popular as a valuable tool in the preoperative assessment of breast masses and it shows high accuracy, sensitivity, and specificity. Differentiating benign from malignant lesions is one of the major goals of FNAC. However, the accuracy of FNAC, with visual interpretation ranges from $65 \%$ to $98 \%$ and is dependent on the doctor's knowledge and experience [6]. Human error would easily cause missed, incorrect or delayed diagnoses. In view of such a situation, computeraided diagnosis technology has been widely applied to reduce a false-negative rate of breast tumor, and increase the rate of true positive [7].

There are currently many computer-aided diagnostic methods for breast cancer. Peng [8] developed a breast cancer diagnosis system from a multi-agent and probabilistic neural network, and used changes in breast tissue resistance to enhance the diagnostic accuracy. Wang [9] used the Learning Vector Quantization (LVQ) neural network model for breast cancer diagnosis and obtained a higher diagnostic accuracy. Jin [10] improved the Back Propagation (BP) neural network for breast cancer diagnosis with additional momentum and adaptive rate. A fuzzy cerebellar model neural network is designed to classify breast nodules with 92.31\% accuracy [11]. A decision tree method was used for breast cancer detection with $94.74 \%$ classification accuracy [12]. A rule induction algorithm was derived from the approximate classification method and applied to a breast 
cancer detection problem achieving $94.99 \%$ accuracy [13]. A neuro-fuzzy technique was proposed, and the accuracy was $95.06 \%$ [14]. A method combining association rules with neural networks $(\mathrm{AR}+\mathrm{NN})$ was proposed for the breast cancer diagnosis problem, and the classification accuracy obtained was $97.4 \%$ [15].

This study proposes a diagnostic method called self-validation cerebellar model articulation controller (SVCMAC) neural network, to distinguish between benign and malignant breast tumors according to intelligent classification. The proposed SVCMAC is a computational model of the human cerebellum [16]. Compared with a neural network, the SVCMAC has the advantages of fast learning, good generalization capability, and simple computation. Moreover, it learns the correct output response to each input vector by modifying the contents of the selected memory locations. Thus, this study used the SVCMAC neural network to classify breast lumps for computer-aided breast tumor diagnosis.

\section{Breast Cancer Diagnosis}

Currently, fine needle aspiration cytology (FNAC) is performed as a pre-operative test to evaluate a suspicious breast lump, where a needle is inserted into the body, and a small amount of tissue is aspirated for examination under a microscope. Then the tissue was identified as benign or malignant through observation and analysis on cell morphological changes and cell qualitative changes [17]. With FNAC becoming more reliable in diagnosing malignancy, the use of frozensection histology had been reduced by about $80 \%$ [18]. However, FNAC has also resulted in missed diagnosis and misdiagnosis because the tissue structure is not observed. Medical research has found a close relationship between tumor characteristics and pathological features, such as lump thickness, uniformity of cell size and cell shape, which were revealed in microscope images of the nucleus of breast tumor tissues.

The Wisconsin breast cancer dataset (WBCD) was collected by Wolberg at the University of Wisconsin-Madison hospitals [19]. The dataset consists of 699 samples taken from fine needle aspirates of human breast tissue, and each sample has nine features: lump thickness, uniformity of cell size, uniformity of cell shape, marginal adhesion, single epithelial cell size, bare nuclei, bland chromatin, normal nucleoli, and mitosis. The measurements are assigned as an integer value between 1 and 10. Each sample has its class label, which is either benign or malignant. 
However, 16 instances were discarded because they contained unavailable value "?", the remaining 683 samples comprised 239 (35\%) malignant and 444 (65\%) benign cases. The SVCMAC neural network can classify the breast tumor according to these nine pathological features to reduce misdiagnoses.

\section{CMAC and SVCMAC Neural Networks}

The Cerebellar Model Articulation Controller (CMAC) is a type of neural network developed from a model of the mammalian cerebellum. The CMAC was first proposed as a function modeler for robotic controllers by Albus in 1975, but has been extensively used in reinforcement learning and also for automated classification in the machine learning community [20]. The CMAC has an associative memory network, and employs error correction learning to drive its memory formation process.

The conventional CMAC, shown in Fig. 1, in general, is trained by presenting pairs of input points and output values, and adjusting the weights in the activated cells by a proportion of the error observed at the output. An input " $X$ " given by the set of vectors so that $X=\left\{x_{1}, x_{2}, x_{3}, x_{4} \ldots x_{n}\right\}$ is mapped to the desired output vector " $Y$ " given by $Y=\left\{y_{1}, y_{2}, y_{3}, y_{4} \ldots y_{n}\right\}$. The mapping function " $F$ " can be given by the following equation:

$$
y_{i}=F\left(x_{1}^{i}, x_{2}^{i}, \cdots, x_{p}^{i}\right)
$$

CMAC can be either a single-input or multiple-input system which utilizes the given mapping function " $F$ " to compute the output. In the case of a multiple-input system where the inputs $x_{1}, x_{2} \ldots x_{n}$ are simultaneously considered, hashing techniques are utilized to generate address table. Hashing can be defined as a technique for obtaining the address table when two or more values are considered as inputs to the CMAC network. The sum of all the weights that the address pointers are pointing towards is equal to the output of the CMAC. After this, the output of CMAC is compared against the pre-determined output value; the training algorithms such as least mean square, gradient decent and back propagation are then executed, and the weights of CMAC are updated till the required minimum error has been achieved at the output. 


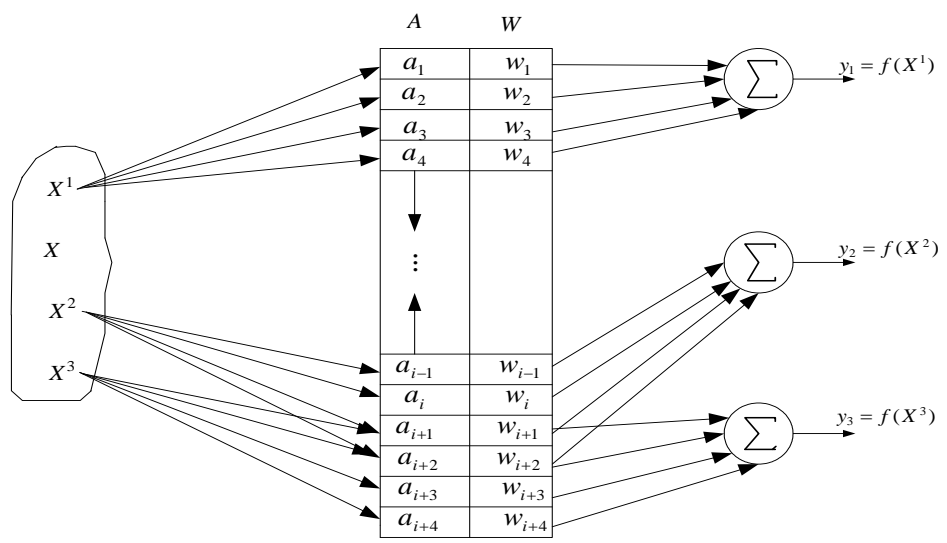

Figure 1

Structure of a CMAC network

This study proposed an advanced self-validation cerebellar model neural network as a classifier. Benign or malignant breast nodules are classified according to the pathological features extracted. Fig. 2 shows an SVCMAC neural network, which is composed of input space, association memory space, receptive- field space, weight memory space, output space, and a self-validation unit. The signal propagation and the basic function in each space are described as follows.

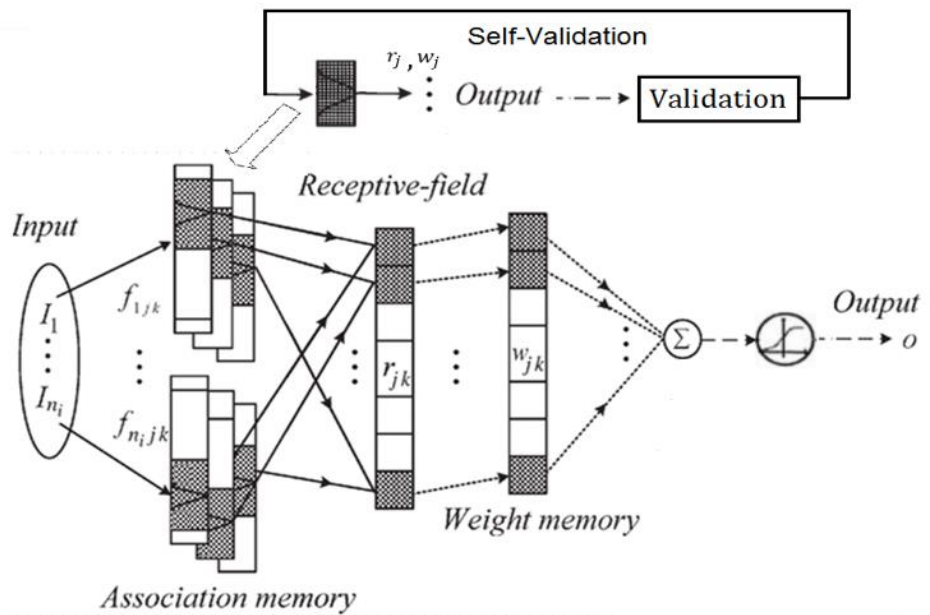

Figure 2

Structure of an SVCMAC network 


\section{1) Forward Computation}

a) The Input space: $I=\left[I_{1}, \cdots, I_{i}, \cdots, I_{n_{i}}\right]^{T} \in \Re^{n_{i}}$, where $n_{i}$ is the number of input state variables, each input state variable, $I_{i}$ can be quantized into discrete regions (called elements or neurons), according to a given control space. The number of elements $n_{e}$ is referred to as the resolution.

b) The Association memory space (Membership function): Several elements can be accumulated as a block. In this space, each block acts as a receptive-field basis function. The Gaussian function is used here as a receptive-field basis function, which can be represented as

$f_{i j k}\left(F_{i j k}\right)=\exp \left(-F_{i j k}^{2}\right)$,

for $i=1,2, \cdots, n_{i}, j=1,2, \cdots, n_{j}$, and $k=1,2, \cdots, n_{k}$

where $F_{i j k}=\frac{I_{i}-m_{i j k}}{v_{i j k}}, m_{i j k}$ is a mean parameter and $v_{i j k}$ is a variance parameter.

c) The Receptive-field space (hypercube): The multi-dimensional receptive-field function is defined as

$$
\begin{array}{r}
r_{j k}=\prod_{i=1}^{n_{i}} f_{i j k}\left(F_{i j k}\right)=\prod_{i=1}^{n_{i}} \exp \left(-\left[\frac{I_{i}-m_{i j k}}{v_{i j k}}\right]^{2}\right), \\
\text { for } \quad j=1,2, \cdots, n_{j}, \text { and } k=1,2, \cdots n_{k}
\end{array}
$$

where $r_{j k}$ is associated with the $j^{\text {th }}$ layer and the $k^{\text {th }}$ block.

d) The Weight memory space $\boldsymbol{w}$ : Each location of a receptive-field to a particular adjustable value in the weight memory space can be expressed as

$$
\begin{aligned}
\boldsymbol{w} & =\left[w_{11}, \cdots, w_{1 n_{k}}, w_{21}, \cdots, w_{2 n_{k}}, \cdots, w_{n_{j} 1}, \cdots, w_{n_{j} n_{k}}\right]^{T} \in \mathfrak{R}^{n_{j} n_{k}} \\
& =\left[w_{1}, \cdots, w_{l}, \cdots, w_{n_{l}}\right]^{T} \in \mathfrak{R}^{n_{l}}
\end{aligned}
$$

where $w_{j k}$ denotes the connecting weight value of the output associated with the $j^{\text {th }}$ layer and the $k^{\text {th }}$ block. 
e) Output space: The output of the SVCMAC is the algebraic sum of the activated weighted receptive-field and is expressed as

$y_{n e t}=u_{S V C M A C}=\boldsymbol{w}^{T} \boldsymbol{r}=\sum_{j=1}^{n_{j}} \sum_{k=1}^{n_{k}} w_{j k} r_{j k}=\sum_{l=1}^{n_{l}} w_{l} r_{l}$

$y_{o}=\frac{1}{1+e^{-y_{n e t}}}$

where $y_{\text {net }}$ is the output of the SVCMAC neural network and $y_{o}$ is the output of the classification.

\section{2) Backward Parameter Adjustment}

a) Back propagation (BP) employed to adjust the parameters is the steepest decent algorithm that has been designed to minimize the error of an objective function defined as:

$$
\begin{aligned}
& e=y_{r e f}-y_{o} \\
& E=\frac{1}{2} \cdot e^{2}
\end{aligned}
$$

where $y_{\text {ref }}$ is the previously known value for the testing set;

$\mathrm{y}_{\text {ref }}=0$ for a malignant lump, and $y_{\text {ref }}=1$ for a benign lump. $m_{i j k}$ and $v_{i j k}$ of the Gaussian function, and output weight $w_{j k}$ are updated respectively as

$$
\begin{aligned}
& w_{j k}(N+1)=w_{j k}(N)+\Delta w_{j k} \\
& m_{i j k}(N+1)=m_{i j k}(N)+\Delta m_{i j k} \\
& v_{i j k}(N+1)=v_{i j k}(N)+\Delta v_{i j k}
\end{aligned}
$$

The updating laws (9) to (11) perform error BP with the following chain rules:

$$
\begin{aligned}
\Delta w_{j k} & =-\eta_{w} \cdot \frac{\partial E}{\partial w_{j k}}=-\eta_{w} \cdot \frac{\partial E}{\partial y_{o}} \cdot \frac{\partial y_{o}}{\partial y_{n e t}} \cdot \frac{\partial y_{n e t}}{\partial w_{j k}} \\
& =\eta_{w} \cdot\left(y_{r e f}-y_{o}\right) \cdot y_{o} \cdot\left(1-y_{o}\right) \cdot r_{j k}=\eta_{w} \cdot e_{p} \cdot r_{j k}
\end{aligned}
$$




$$
\begin{aligned}
\Delta m_{i j k} & =-\eta_{m} \cdot \frac{\partial E}{\partial m_{i j k}}=-\eta_{m} \cdot \frac{\partial E}{\partial y_{o}} \cdot \frac{\partial y_{o}}{\partial y_{n e t}} \cdot \frac{\partial y_{n e t}}{\partial r_{j k}} \cdot \frac{\partial r_{j k}}{\partial m_{i j k}} \\
& =2 \cdot \eta_{m} \cdot e_{p} \cdot w_{j k} \cdot r_{j k} \cdot \frac{x_{i}-m_{i j k}}{v_{i j k}^{2}} \\
\Delta v_{i j k} & =-\eta_{v} \cdot \frac{\partial E}{\partial v_{i j k}}=-\eta_{v} \cdot \frac{\partial E}{\partial y_{o}} \cdot \frac{\partial y_{o}}{\partial y_{n e t}} \cdot \frac{\partial y_{n e t}}{\partial r_{j k}} \cdot \frac{\partial r_{j k}}{\partial v_{i j k}} \\
& =2 \cdot \eta_{v} \cdot e_{p} \cdot w_{j k} \cdot r_{j k} \cdot \frac{\left(x_{i}-m_{i j k}\right)^{2}}{v_{i j k}^{3}}
\end{aligned}
$$

Where,

$$
e_{p}=\left(y_{r e f}-y_{o}\right) \cdot y_{o} \cdot\left(1-y_{o}\right)
$$

Among these, $\eta_{w}, \eta_{m}$ and $\eta_{v}$ are positive learning rates for $w_{k}, m_{i j k}$ and $v_{i j k}$, respectively.

\section{b) Self-validation algorithm}

The training process will be terminated on any one of the following three conditions. First, the error of the sample is not decreased for six consecutive iterations; second, the error is equal to zero; and third, the maximum number of epochs is reached.

The data used in this study are divided into three categories, training data, validation data and test data. The training data with initial values of $w_{j k}, m_{i j k}$ and $v_{i j k}$ being random are employed to train the SVCMAC neural network, while the validation data test and verify the neural network. After training, $w_{j k}, m_{i j k}$ and $v_{i j k}$ are updated and serve as a self-validation unit to start a new SVCMAC training epoch with the same training data set and validation data in order to improve the accuracy rate. After 100 such iterations, the value of three parameters that attain the highest accuracy rate on the validation data set are saved, and finally employed to classify the test data. Fig. 3 summarizes the SVCMAC training procedure. 


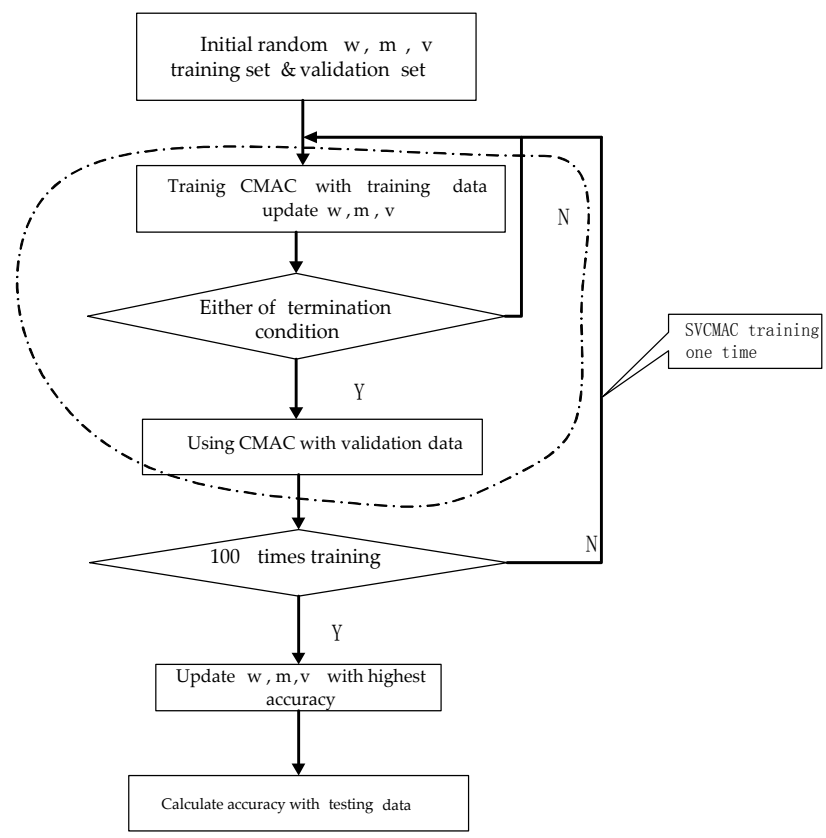

Figure 3

The procedure of the SVCMAC training

\section{Performance Evaluation}

The performance evaluation of the proposed method is carried out using the random data selection approach. The dataset is randomly divided into three subsets; that is, $50 \%$ of the data are for training, $30 \%$ for validation, and $20 \%$ for testing. The performance of the proposed SVCMAC neural network classification method is evaluated with sensitivity, specificity and accuracy tests. Sensitivity, specificity and accuracy terms are commonly used statistics, which uses the True positive (TP), true negative (TN), false negative (FN), and false positive (FP) terms. TP is number of true positives, denoting cases in the 'positive' class that are correctly classified as positive; FP, number of false positives, denoting cases in the 'positive' class that are misclassified as positive, and should be in the 'negative class'; TN, number of true negatives, denoting cases in the 'negative' 
class that are correctly classified as negative; and FN, number of false negatives, denoting cases in the 'negative' class that should be classified as positive. Thus, sensitivity, specificity and accuracy are described in the following equations:

$$
\begin{aligned}
& \text { Accuracy }(A C C)=(T P+T N) /(T N+T P+F P+F N) \times 100 \% \\
& \text { Sensitivity }(S E N)=T P /(T P+F N) \times 100 \% \\
& \text { Specificity }(S P E)=T N /(T N+F P) \times 100 \%
\end{aligned}
$$

$T P$ : Malignant case identified as malignant.

FP: Benign case identified as malignant.

$T N$ : Benign case identified as benign.

FN: Malignant case identified as benign.

\section{Experiment and Results}

The WBCD with nine attributes and 683 records was used in the experiment. The parameters for the SVCMAC neural network, including weight $(w)$, mean $(m)$, and variance $(v)$, are also randomly initialized. The samples are randomly selected for training and testing datasets. In the end, there were 341 samples in the training dataset; 205 data were used for validation and the remaining 137 samples were for testing. The training will run 1000 iterations but can stop any time when the accuracy rate is $100 \%$. The updated $w, m$ and $v$ of validation data serve as the selfvalidation unit of the SVCMAC, which provide new values to the next SVCMAC training epoch. After 100 iterations, the $w, m, v$ were updated with the highest accuracy rate of validation data set.

Back Propagation neural network (BP), Learning Vector Quantization Neural network (LVQ), CMAC neural network and SVCMAC neural network were all employed to train and identify breast cancer from the same data sets. The training set contained 541 sets of data, 352 benign and 189 malignant cases; and the testing set comprised 142 sets of data, 92 benign and 50 malignant cases. It should be mentioned that the SVCMAC neural network needs the validation set only. The original training set is divided into two sets, the training set containing 341 sets of data with 220 benign and 121 malignant cases, and the validation set comprising 200 sets of data with 132 benign and 68 malignant cases. Table 1 shows the simulation results of BP, LVQ, CMAC and SVCMAC. 
Table 1

Classification with $80 \%$ training data and $20 \%$ testing data

\begin{tabular}{|c|c|c|c|c|c|}
\hline \multicolumn{2}{|r|}{ Methods } & BP & LVQ & CMAC & SVCMAC \\
\hline \multirow[t]{3}{*}{ Acc } & Highest & $97.81 \%$ & $97.08 \%$ & $96.35 \%$ & $98.12 \%$ \\
\hline & Lowest & $87.59 \%$ & $93.43 \%$ & $93.43 \%$ & $94.53 \%$ \\
\hline & Avg & $93.72 \%$ & $94.96 \%$ & $95.03 \%$ & $96.5 \%$ \\
\hline \multirow[t]{3}{*}{ Sen } & Highest & $96.23 \%$ & $92.68 \%$ & $96.52 \%$ & $98.5 \%$ \\
\hline & Lowest & $69.57 \%$ & $84 \%$ & $87.5 \%$ & $93.35 \%$ \\
\hline & $\overline{\mathrm{Avg}}$ & $84.7 \%$ & $87.5 \%$ & $91.49 \%$ & $95.16 \%$ \\
\hline \multirow[t]{3}{*}{ Spe } & Highest & $100 \%$ & $100 \%$ & $98.89 \%$ & $99.1 \%$ \\
\hline & Lowest & $94.05 \%$ & $97.7 \%$ & $93.4 \%$ & $96.65 \%$ \\
\hline & $\overline{~ A v g}$ & $97.63 \%$ & $99 \%$ & $97.78 \%$ & $97.85 \%$ \\
\hline
\end{tabular}

All the algorithms are executed with 10 folds.

Avg=average

Analyzing the diagnosis of BP, LVQ, CMAC and SVCMAC revealed that the SVCMAC neural network has higher diagnosis accuracy than the LVQ, BP and CMAC. As shown in Table 1, the SVCMAC neural network has higher sensitivity, which can assist physicians in making early and correct diagnosis on breast cancer, and can reduce misdiagnoses at the same time. In summary, the SVCMAC which has a higher diagnostic accuracy and lower false-negative rate is a reliable method for the computer-aided diagnosis of breast cancer.

Table 2

Classification accuracies for each fold

\begin{tabular}{||c||c||c||c||c||c||}
\hline Folds & $\begin{array}{c}\text { Number of } \\
\text { training data }\end{array}$ & $\begin{array}{l}\text { Number of } \\
\text { test data }\end{array}$ & $\begin{array}{l}\text { Correct } \\
\text { classified }\end{array}$ & $\begin{array}{c}\text { Miss } \\
\text { classified }\end{array}$ & $\begin{array}{c}\text { Correct classification } \\
\text { rate }(\%)\end{array}$ \\
\hline \hline 1 & 541 & 142 & 136 & 6 & $95.7 \%$ \\
\hline \hline 2 & 541 & 142 & 138 & 4 & $97.2 \%$ \\
\hline \hline 3 & 541 & 142 & 137 & 5 & $96.4 \%$ \\
\hline \hline 4 & 560 & 123 & 120 & 3 & $97.2 \%$ \\
\hline \hline 5 & 560 & 123 & 119 & 4 & $96.7 \%$ \\
\hline
\end{tabular}

Moreover, a five-fold cross validation test was applied and the average values were calculated for performance measurements. In the cross validation test, the first three folds contained 541samples for the training dataset with the remaining 142 samples for testing; and in the last two folds, the training set contained 560 samples with the remaining 123 samples for testing. Table 2 lists the accuracy 
values calculated for each fold with correct classification rates and the number of misclassified samples. As can be seen, the best performance is obtained in the second and fourth folds with calculated accuracy exceeding $97 \%$.

\section{Conclusions}

This study proposed a SVCMAC neural network algorithm for application in breast cancer diagnosis. Compared with current breast cancer diagnosis approaches, the proposed SVCMAC neural network classifier achieves a higher accuracy rate. The textural feature method overcomes the natural drawbacks of FNAC. Hence, the SVCMAC neural network is most suitable for classifying WBCD data and is very helpful to oncologists in making the ultimate diagnosis decision.

\section{Acknowledgment}

This work was supported by Natural Sciences and Engineering Research Council of Canada, National Natural Science Foundation of China (Nos. 61573296, and 61473329), the Specialized Research Fund for the Doctoral Program of Higher Education of China (No.20130121130004), and the Fundamental Research Funds for the Central Universities in China (Xiamen University: No. 2013121025, No. 201412G009, and No. 2014X0234), the National Science Council of the Republic of China under Grant NSC-95-2221-E-155-014-MY3.

\section{References}

[1] World Cancer Report 2014, World Health Organization. 2014, Chapter1.1

[2] World Cancer Report 2014, World Health Organization. 2014, Chapter5.2

[3] World Health Organization (2008) World Cancer Report, IARC Press

[4] http://www.wcrf.org/int/cancer-facts-figures/data-specific-cancers/breastcancer-statistics http://www.wcrf.org/int/cancer-facts-figures/dataspecific-cancers/breast-cancer-statistics

[5] A. N. Pandya and N. P. Shah, "Breast Fine Needle Aspiration Cytology Reporting: a Study of Application of Probabilistic Approach," Indian Medical of Gazette, Vol. 147, No. 2, pp. 54-59, 2013

[6] A. Muratli, N. Erdogan, S. Sevim, I. Unal and S. Akyuz "Diagnostic Efficacy and Importance of Fine-Needle Aspiration Cytology of Thyroid Nodules," Journal of Cytology, Vol. 31, No. 2, pp. 73-78, 2014

[7] R. A. Castellino, "Computer-aided Detection (CAD): an Overview," Cancer Imaging, Vol. 5, No. 1, pp. 17-19, 2005 
[8] Y. Peng and Y. Q. Chen, "Diagnosis System of Breast Cancer based on Probabilistic Neural Network," Journal of Hefei University of Technology, Vol. 36, No. 6, pp. 685-687, 2013

[9] B. Wang, X. X. Du and M. Jin, "Application of Breast Tumor Diagnosis based on Learning Vector Quantization Neural Network," Journal of Computer Simulation, Vol. 29, no. 8, pp. 171-174, 2012

[10] Q. Jin and P. Z. Gao, "Study on Breast Cancer Diagnosis based on Artificial Neural Network," Journal of Computer Simulation, Vol. 28, No. 6, pp. 235238,2011

[11] C. M. Lin, Y. L. Hou, T. Y. Chen and K. H. Chen, "Breast Nodules Computer-aided Diagnostic System Design Using Fuzzy Cerebellar Model Neural Networks," IEEE Transactions on Fuzzy Systems, Vol. 22, No. 3, pp. 693-699, 2014. C. M. Lin, Y. L. Hou, T. Y. Chen and K. H. Chen, "Breast Nodules Computer-aided Diagnostic System Design using Fuzzy Cerebellar Model Neural Networks," IEEE Transactions on Fuzzy Systems, Vol. 22, No. 3, pp. 693-699, 2014

[12] J. R. Quinlan, "Improved Use of Continuous Attributes in C4. 5," Journal of Artificial Intelligence Research, Vol. 4, pp. 77-90, 1996

[13] H. J. Hamilton, N. Shan and N. Cercone, "RIAC: a Rule Induction Algorithm based on Approximate Classification," Technical Report CS 9606, University of Regina, 1996. H. J. Hamilton, N. Shan and N. Cercone, "RIAC: a Rule Induction Algorithm based on Approximate Classification," Technical Report CS 96-06, University of Regina, 1996

[14] D. Nauck and R. Kruse, "Obtaining Interpretable Fuzzy Classification Rules from Medical Data," Artificial Intelligence in Medicine, Vo1. 6, No. 2, pp. 149-169, 1999

[15] M. Karabatak and M. C. Ince, "An Expert System for Detection of Breast Cancer based on Association Rules and Neural Network," Expert Systems with Applications, Vol. 36, No. 2, part 2, pp. 3465-3469, 2009

[16] W. Li, Y. Li, W. Yang, Q. Zhang, D. Wei and W. Li, “ Brain Structures and Functional Connectivity Associated with Individual Differences in Internet Tendency in Healthy Young Adults," Neuropsychologia, Vol. 70, pp. 134144,2015

[17] A. O. Daramola, M. O. Odubanjo, F. J. Obiajulu and N. Z. Ikeri "Correlation between Fine-Needle Aspiration Cytology and Histology for Palpable Breast Masses in a Nigerian Tertiary Health Institution," International Journal of Breast Cancer, article id 742573, 2015 
[18] MZ. Rahman, AM. Sikder and SR. Nabi, "Diagnosis of Breast Lump by Fine Needle Aspiration Cytology and Mammography," Mymensingh Medical Journal, Vol. 20, No. 4, pp. 658-664, 2011

[19] A. Marcano-Cedeño, J. Quintanilla-Domíngueza, and D. Andina, "WBCD Breast Cancer Database Classification Applying Artificial Metaplasticity Neural Network," Expert Systems with Applications, Vol. 38, No. 8, pp. 9573-9579, 2011

[20] J. S. Albus, "A New Approach to Manipulator Control: the Cerebellar Model Articulation Controller (CMAC)," Journal of Dynamic Systems, Measurement and Control, Vol. 97, No. 3, pp. 220-227, 1975. J. S. Albus, "A New Approach to Manipulator Control: the Cerebellar Model Articulation Controller (CMAC)," Journal of Dynamic Systems, Measurement and Control, Vol. 97, No. 3, pp. 220-227, 1975

[21] J. S. Albus, "Mechanisms of Planning and Problem Solving in the Brain," Mathematical Biosciences, Vol. 45, No. 3, pp. 247-293, 1979

[22] C. M. Lin, Y. M. Chen and C. S. Hsueh, "A Self-Organizing Interval Type-2 Fuzzy Neural Network for Radar Emitter Identification," International Journal of Fuzzy Systems, Vol. 16, No. 1, pp. 20-30, 2014

[23] C. M. Lin, C. F. Tai and C. C. Chung, "Intelligent Control System Design for UAV Using a Recurrent Wavelet Neural Network," Neural Computing and Applications, Vol. 24, No. 2, pp. 487-496, 2014

[24] C. M. Lin and H. Y. Li, "TSK Fuzzy CMAC-based Robust Adaptive Backstepping Control for Uncertain Nonlinear Systems," IEEE Transaction on Fuzzy Systems, Vol. 20, No. 6, pp.1147-1154, $2012 . \quad$ C. M. Lin and H. Y. Li, "TSK Fuzzy CMAC-based Robust Adaptive Backstepping Control for Uncertain Nonlinear Systems," IEEE Transaction on Fuzzy Systems, Vol. 20, No. 6, pp. 1147-1154, 2012

[25] S. D. Teddy, "KCMAC: a Novel Fuzzy Cerebellar Model for Medical Decision Support," Artificial Neural Networks-ICANN 2008, Vol. 5164, pp. 537-546, 2008

[26] H. A. Khan, A. C. M. Tan and Y. Xiao, "An Implementation of Novel CMAC Algorithm for Very Short Term Load Forecasting," Journal of Ambient Intelligence and Humanized Computing, Vol. 4, No. 6, pp. 673683, Dec 2013

[27] C. M. Lin and C. H. Chen, "Robust Fault-Tolerant Control for a Biped Robot Using a Recurrent Cerebellar Model Articulation Controller," IEEE Transactions on Systems, Man, and Cybernetics, Vol. 37, No. 1, pp. 110123, 2007 